\title{
Four-particle condensate in strongly coupled fermion systems
}

\author{
G. Röpke, A. Schnell, P. Schuck* \\ University of Rostock, FB Physik, Universitätsplatz 1, 18051 Rostock, Germany \\ P. Nozières \\ Institut Laue-Langevin, B.P. 156, 38042 Grenoble Cedex 9, France
}

(October 15, 2018)

\begin{abstract}
Four-particle correlations in fermion systems at finite temperatures are investigated with special attention to the formation of a condensate. Instead of the instability of the normal state with respect to the onset of pairing described by the Gorkov equation, a new equation is obtained which describes the onset of quartetting. Within a model calculation for symmetric nuclear matter, we find that below a critical density, the four-particle condensation ( $\alpha$-like quartetting) is favored over deuteron condensation (triplet pairing). This pairing-quartetting competition is expected to be a general feature of interacting fermion systems, such as the excition-biexciton system in excited semiconductors. Possible experimental consequences are pointed out.

Keywords: Bose-Einstein condensation, superfluidity, strongly coupled systems, nuclear matter, $\alpha$-particle matter, exciton-biexciton system.
\end{abstract}

05.30.Fk, 03.75.F, 67.60, 21.65

Typeset using REVTEX 
One of the most amazing phenomena in quantum many-particle systems is the formation of quantum condensates. Of particular interest are strongly coupled fermion systems where bound states arise. In the low-density limit, where even-number fermionic bound states can be considered as bosons, Bose-Einstein condensation may be expected to occur at low temperatures. At present, condensates are investigated in systems where the cross-over from Bardeen-Cooper-Schrieffer (BCS) pairing to Bose-Einstein condensation (BEC) can be observed, see [1]. Among very different quantum systems such as electron-hole exciton system in semiconductors, atoms in traps at extremely low temperatures, etc., nuclear matter is particularly suited to study correlation effects in a quantum liquid.

An indication of strong correlations in nuclear matter is the formation of bound states. The interaction in the singlet $(S=0)$ channel is not strong enough to form a bound state, whereas in the neutron $(\mathrm{n})$ - proton $(\mathrm{p})$ triplet $(S=1)$ channel a bound state, the deuteron, arises. In nuclear matter, with increasing density the bound states are modified and will disappear at the so-called Mott density [2]. In particular, treating the two-particle Green function in ladder Hartree-Fock approximation, an effective wave equation (in matrix notation) $\psi_{\lambda}=K_{2}\left(E_{\lambda}\right) \psi_{\lambda}$ for the quantum state $\lambda$ can be derived. Explicitly this reads

$$
\psi_{\lambda}(12)=\sum_{1^{\prime} 2^{\prime}} K_{2}\left(12,1^{\prime} 2^{\prime}, E_{\lambda}\right) \psi_{\lambda}\left(1^{\prime} 2^{\prime}\right)
$$

with

$$
K_{2}\left(12,1^{\prime} 2^{\prime}, z\right)=V\left(12,1^{\prime} 2^{\prime}\right) \frac{1-f(1)-f(2)}{z-E(1)-E(2)} .
$$

The influence of the medium is contained in the single-particle energy $E(1)=p_{1}^{2} / 2 m+$ $\sum_{2} V(12,12)_{\mathrm{ex}} f(2)$ and in the Pauli blocking term $[1-f(1)-f(2)]$. Here $f(1)=$ $[\exp \{E(1) / T-\mu / T\}+1]^{-1}$ is the Fermi distribution function and '1' stands for momentum, spin, and isospin coordinates, whereas $V\left(12,1^{\prime} 2^{\prime}\right)$ is the antisymmetrized matrix element of the two-body interaction.

Including medium modifications of two-particle states, a generalized Beth-Uhlenbeck formula $N / \Omega_{0}=n_{1}(T, \mu)+n_{\text {corr }}(T, \mu)$ has been derived [3, [4], where the uncorrelated density 
$n_{1}(T, \mu)=\Omega_{0}^{-1} \sum_{1} f(1)$ is given by the quasi-particle contribution $\left(\Omega_{0}\right.$ being the normalization volume). The correlated density $n_{\text {corr }}(T, \mu)$ is obtained from the two-particle T matrix. This approach has been widely applied to ionic plasmas as well as to the electron-hole exciton system in excited semiconductors [5]. Taking into account the Mott effect, general features of the composition of nuclear matter as function of density and temperature are given in [6].

At low temperatures it is well known that in nuclei, nuclear matter and neutron matter (neutron stars) superfluidy can arise in the singlet channel [7 9]. A theoretical description of this superfluidity can be achieved by treating the Gorkov equation $\psi_{2}=K_{2}\left(\mu_{1}+\mu_{2}\right) \psi_{2}$ for the critical temperature $T_{2}^{c}$ as a function of the chemical potential. It allows the determination of $T_{s}^{c}$ or $T_{t}^{c}$ for the singlet and triplet channels, respectively.

The solution of the Gorkov equation has been considered by different authors using realistic nucleon-nucleon interactions. It has been found that in comparison with the singlet channel, in the triplet channel the transition to superfluidity should arise at relatively high temperatures [10,11]. This is a consequence of the stronger interaction in the triplet channel which leads to the formation of a bound state (deuteron) in the low-density limit where $f \ll 1$. Estimates give a value of the critical temperature up to $T_{t}^{c} \approx 5 \mathrm{MeV}$ at one third of the nuclear matter density. At the same time, at zero temperature a large gap arises [11. An interesting feature of the triplet pairing in symmetric nuclear matter is the cross-over from Bose-Einstein condensation of deuterons at low densities to BCS neutron-proton pairing at high densities [6].

In spite of the relatively strong interaction, cf. also calculations with effective pairing forces by Goodman [12], triplet pairing seems less apparent in nuclear structure systematics 13. However, it should become important for heavier $N=Z$ nuclei produced in the new radioactive beam facilities.

In this letter we show that in the low-density region the transition to triplet pairing is not realized, because four-particle correlations are more dominant there. Obviously, at 
chemical equilibrium, in the low-density region at low temperatures the dominant part of nuclear matter will be found in $\alpha$-particles which are much stronger bound than the deuteron. Therefore, the triplet pairing (Bose condensation of deuterons) has to compete with quartetting (Bose condensation of $\alpha$-particles).

The four-particle correlations are obtained from the four-particle Green function (compare [14]) that is given in ladder Hartree-Fock approximation by the equation

$$
\begin{aligned}
& G_{4}\left(1234,1^{\prime} 2^{\prime} 3^{\prime} 4^{\prime}, z\right)=\frac{f(1) f(2) f(3) f(4)}{g_{4}(1234)} \frac{\delta_{11^{\prime}} \delta_{22^{\prime}} \delta_{33^{\prime}} \delta_{44^{\prime}}}{z-E_{4}(1234)} \\
& +\sum_{1^{\prime \prime} 2^{\prime \prime} 3^{\prime \prime} 4^{\prime \prime}} K_{4}\left(1234,1^{\prime \prime} 2^{\prime \prime} 3^{\prime \prime} 4^{\prime \prime}, z\right) G_{4}\left(1^{\prime \prime} 2^{\prime \prime} 3^{\prime \prime} 4^{\prime \prime}, 1^{\prime} 2^{\prime} 3^{\prime} 4^{\prime}, z\right)
\end{aligned}
$$

where we use the abbreviation $E_{n}(12 \ldots n)=E(1)+E(2)+\cdots+E(n)$, and $g_{n}(12 \ldots n)=$ $\left[\exp \left(E_{n}(12 \ldots n)-n \mu\right) / T-1\right]^{-1}$ being the Bose distribution function.

The instantaneous part of interaction kernel is obtained by using the technique of Matsubara Green functions as

$$
\begin{aligned}
K_{4}\left(1234,1^{\prime} 2^{\prime} 3^{\prime} 4^{\prime}, z\right) & =V\left(12,1^{\prime} 2^{\prime}\right) \frac{f(1) f(2)}{g_{2}(12)} \frac{\delta_{33^{\prime}} \delta_{44^{\prime}}}{z-E_{4}(1234)} \\
& + \text { perm. }
\end{aligned}
$$

where the terms obtained by renumbering are not given explicitly. We have used the identity $\bar{f}(1) \bar{f}(2) \cdots \bar{f}(n)-f(1) f(2) \cdots f(n)=g_{n}^{-1}(12 \ldots n) f(1) f(2) \cdots f(n)$ with $\bar{f}=1-f$.

Near a pole the Green function $G_{4}$ can be factorized, $G_{4}\left(1234,1^{\prime} 2^{\prime} 3^{\prime} 4^{\prime}, z\right) \approx$ $\psi_{\nu}(1234) \psi_{\nu}^{*}\left(1^{\prime} 2^{\prime} 3^{\prime} 4^{\prime}\right) /\left(z-E_{\nu}\right)$. The eigenvalues $E_{\nu}$ and eigenstates $\psi_{\nu}(1234)$ follow from the solution of the four-particle Schrödinger-like wave equation $\psi_{\nu}=K_{4}\left(E_{\nu}\right) \psi_{\nu}$. The eigenvalue $E_{0}(T, \mu)$ of the lowest bound state ( $\alpha$-particle) depends on temperature $T$ and chemical potential $\mu$ and also on its center-of-mass momentum due to the medium-dependent self-energy as well as the phase space occupation factors.

From the four-particle Green function, the four-particle density matrix is obtained as

$$
\begin{aligned}
& <a_{1}^{+} a_{2}^{+} a_{3}^{+} a_{4}^{+} a_{4^{\prime}} a_{3^{\prime}} a_{2^{\prime}} a_{1^{\prime}}> \\
& =\int \frac{d \omega}{\pi} g_{4}(\omega) \operatorname{Im} G_{4}\left(1234,1^{\prime} 2^{\prime} 3^{\prime} 4^{\prime}, \omega-i 0\right) .
\end{aligned}
$$


Obviously, the four-particle density (5) diverges when, at a given temperature $T$, the chemical potential takes the value $\mu_{c}=E_{0}\left(T, \mu_{c}\right) / 4$. Then, the delta function produced by the pole of $G_{4}$ coincides with the singularity of the Bose function. Values for the chemical potential $\mu$ exceeding the value of the lowest bound state energy $E_{0}(T, \mu)$ are not admissible because the diagonal elements of the four-particle density are positive definite.

The main objective of this letter is to give an estimate of the critical temperature $T_{4}^{c}(\mu)$ for the onset of a four-particle condensate. This is obtained by solving the equation

$$
\psi_{4}(1234)=\sum_{1^{\prime} 2^{\prime} 3^{\prime} 4^{\prime}} K_{4}\left(1234,1^{\prime} 2^{\prime} 3^{\prime} 4^{\prime}, 4 \mu\right) \psi_{4}\left(1^{\prime} 2^{\prime} 3^{\prime} 4^{\prime}\right)
$$

In the low-density limit, where the distribution functions occurring in $K_{4}$ are small compared with 1, this equation coincides with the Schrödinger equation for the four-particle bound state, i.e. the $\alpha$-particle in free space.

To discuss the competition between two-particle pairing and the condensation of four-particle states, we perform an exploratory calculation for a simple model system which contains the formation of a two-particle bound state in the triplet channel and a singlet four-particle bound state. We use a separable interaction of Gaussian form $V_{s / t}\left(12,1^{\prime} 2^{\prime}\right)=-\lambda_{s / t} \Omega_{0}^{-1} \exp \left(-p_{12}^{2} / b^{2}\right) \exp \left(-p_{12}^{\prime 2} / b^{2}\right) \delta\left(q_{12}-q_{12}^{\prime}\right)$ with the relative momentum $p_{12}=\left(p_{1}-p_{2}\right) / 2$ and the center-of-mass momentum $q_{12}=p_{1}+p_{2}$. At given range parameter $b=1.54 \mathrm{fm}^{-1}$, the interaction strengths are adopted as $\lambda_{t}=1213.8 \mathrm{MeVfm}^{3}$ and $\lambda_{s}=536 \mathrm{MeVfm}^{3}$ to reproduce the free deuteron binding energy $E_{t}=-2.225 \mathrm{MeV}$ in the neutron-proton triplet channel as well as the free $\alpha$ binding energy $E_{0}=-28.29 \mathrm{MeV}$ for the four-particle ground state (see below). In principle it is possible to extend the calculations to more realistic nucleon-nucleon potentials as given in 15 .

We begin with the discussion of neutron-proton triplet pairing. The critical temperature $T_{t}^{c}$ obtained from the solution of the Gorkov equation is presented as a function of the reduced chemical potential $\mu^{*}$ in Fig. 1 and as a function of the uncorrelated density $n_{1}(T, \mu)=$ $\Omega_{0}^{-1} \sum_{1} f(1)$ in Fig. 2 (dotted lines). For the sake of simplicity the self-energy shift is taken to be a constant that is incorporated into a shift of the chemical potential, $\mu^{*}=$ 
$\mu-\sum_{2} V(12,12)_{\mathrm{ex}} f(2)$, at $p_{1}=0$.

The four-particle wave equation (3) is solved within a variational ansatz where the fourparticle wave function with zero total momentum is given as a product of two wave functions for the relative motion (quasi deuterons) $\phi(p)$ and the center-of-mass wave function $\psi(q)$,

$$
\psi_{4}(1234)=\phi\left(p_{12}\right) \phi\left(p_{34}\right) \psi\left(q_{12}\right) \delta_{q_{12}+q_{34}, 0}
$$

The wave function for the relative motion $\phi(p)$ is optimized in a space of functions containing the exact solution for the wave function of the in-medium deuteron (Eqs. (11,2)), whereas for the center-of-mass wave function $\psi(q)$ a simple Gaussian form is adopted.

The results for the critical temperature $T_{4}^{c}$ as a function of the chemical potential $\mu^{*}$ and the uncorrelated density $n$ are displayed in Figs. 1 and 2, respectively (solid lines). In the low-temperature limit, with increasing chemical potential the transition to quartetting occurs prior to the pairing transition. This is a consequence of the fact that the value $E_{0} / 4$ for the four-particle bound state lies below the value $E_{t} / 2$ of the triplet bound state. The Gorkov equation, which predicts a normal state for temperatures $T>T_{t}^{c}$, is not applicable in that region, because already at a higher temperature $T_{4}^{c}$ the normal state was removed due to the onset of quartetting.

A simple argument for the behavior of $T_{4}^{c}, T_{t}^{c}$ as a function of $n_{1}$ in the limit of low densities can be given from the law of mass action. Neglecting medium corrections the critical temperatures $T_{t}^{c}$ and $T_{4}^{c}$ as functions of $n_{1}$ are found as solutions of $n_{1}=4\left(m T_{t}^{c} / 2 \pi \hbar^{2}\right)^{3 / 2} \exp \left\{E_{t} / 2 T_{t}^{c}\right\}$ and $n_{1}=4\left(m T_{4}^{c} / 2 \pi \hbar^{2}\right)^{3 / 2} \exp \left\{E_{0} / 4 T_{4}^{c}\right\}$, respectively.

With increasing density the critical temperature $T_{4}^{c}$ approaches $T_{t}^{c}$ from above at a critical density $n_{1}^{0}=0.03 \mathrm{fm}^{-3}\left(\mu_{0}^{*} \approx 10 \mathrm{MeV}\right)$. For $n_{1}<n_{1}^{0}\left(\mu^{*}<\mu_{0}^{*}\right)$ the four-particle condensate arises whereas for $n_{1}>n_{1}^{0}$ the system goes over to a BCS pairing state.

The fact that at higher densities $\alpha$-particle condensation disappears faster than deuteron Cooper pairing is at first sight quite astonishing because of the very strong binding of $\alpha$ 's in free space. This feature, one of the main results of our work, can, however, be explained qualitatively with simple arguments and is generic for any Fermi system where pairing and 
quartetting may interfere. Indeed the competition between pair and quartet condensation in attractive Fermi liquids is known also from the field of semiconductors, where both exciton and biexciton condensates have been suggested. The qualitative features of that competition are easily understood. Since they do not depend crucially on the triplet nature of the condensate we discuss the simpler case of singlet pairing as a function of density. In the weak coupling limit (high density), the ground state is described by the standard BCS wave function. However weak the attraction is, a pairing amplitude $\langle a a\rangle$ appears due to the finite density of states at Fermi level for pairs with zero total momentum $q$ (the two-particle kernel has a logarithmic singularity). In contrast a quartet condensate $\langle$ aaaa $\rangle \neq 0$ does not exist on its own. Due to the exclusion principle the corresponding density of states for $q=0$ vanishes at Fermi level and it takes a minimum attraction to develop a Cooper pole.

In the opposite limit of dilute systems with attraction strong enough to bind individual pairs and quartets, an atomic regime prevails. Single pairs $\langle a a\rangle$ may be viewed as bosons $\langle\Phi\rangle$ and the quartet as a bound pair of two bosons. The competition between particle and pair condensation in Bose liquids was discussed in [16]. In the dilute limit we expect "molecular" Bose condensation of bound bosons, as described long ago by Valatin and Butler [17]. In the dense limit the quartets dissociate and single boson condensation takes over. As the density decreases, a variational Ansatz [16] shows that the condensate fraction $n_{0}=|\langle\Phi\rangle|^{2}$ goes down, vanishing at a critical $n_{c}$ beyond which only $\langle\Phi \Phi\rangle$ survives $\left(n_{c}\right.$ also marks the appearance of a gap).

Altogether, we expect a quartet atomic condensate for very low densities and a BCS pair condensate for very high densities. In between the interpolation depends on the pair and quartet binding energies, $E_{2}$ and $2 E_{2}+E_{4}$, as compared to the Fermi energy. If $E_{2} \gg E_{4}$ the pair condensate enters the atomic regime well before it disappears in favor of quartets. If on the other hand $E_{2}<E_{4}$ the system goes directly from a BCS pair superfluid to a quartet Bose-Einstein condensation.

Possible signatures of quartetting have been discussed in the past in the context of nuclear structure [18]. According to the present studies quartet correlations may be of importance 
in the outer regions of nuclei. We can make a rough estimate for the critical temperature of $\alpha$-particle condensation in nuclei in converting $T_{4}^{c}(n)$ of Fig. 2 via the Local Density Approximation into a radius dependence $T_{4}^{c}(r)$ and averaging over a typical nuclear density. This procedure applied to ordinary neutron-neutron pairing yields $T_{c} \approx 1 \mathrm{MeV}$ in good agreement with more microscopic calculations [19]. In this way we predict that the increase of the critical temperature due to $\alpha$ - particle condensation is about $0.2 \mathrm{MeV}$. In spite of our limited variational approach, we think that this value should be correct within 30 to $40 \%$. Also the critical density $n_{1}^{0}=0.03 \mathrm{fm}^{-3}$ for the onset of quartetting should be reliable within these limits and therefore our estimate can be of interest for clustering phenomena in stellar collapse and the outer crust of neutron stars [9]. We believe that in the far tail of nuclear densities $\left(n<n_{1}^{0}\right)$ quartetting can occur as well as in a number of nuclear processes such as the emission of $\alpha$-particles from the heaviest elements [20] and unusually large $\alpha$-decay rates of neutron deficient lead isotopes [21]. Bose-Einstein effects may also be of relevance in heavy-ion collisions at a relatively late stage where the temperature of expanding matter has dropped below $5 \mathrm{MeV}$.

In conclusion, we have shown that prior to the transition to pairing, a transition from the normal state to four-particle condensation according to Eq. (66) can occur. However, as a generic phenomenon 4-body condensation always looses against weak coupling Cooper pairing at sufficiently high densities. For demonstration purposes we have considered nuclear matter as a strongly coupled quantum liquid. The possibility of quartetting and higher correlated condensates is of interest also in other systems, e.g., electron-hole pairs in semiconductors forming bi-excitons [1,22] or quarks forming two-pion states [23].

G. R. and A. S. appreciate useful discussions with G. Bertsch. P. S. is grateful to Deutsche Forschungsgemeinschaft (DFG) for financial support and to University of Rostock for pleasant hospitality. 


\section{REFERENCES}

* Permanent Address: Institut de Sciences Nucléaires, Université Joseph Fourier, CNRSIN2P3 53, Avenue des Martyrs, F-38026 Grenoble Cedex, France

[1] A. Griffin, D. W. Snoke, and S. Stringari (eds.), Bose-Einstein Condensation (Cambridge University Press, Cambridge, 1995).

[2] G. Röpke, L. Münchow, and H. Schulz, Nucl. Phys. A379, 536 (1982); Phys. Lett. B 110, 21 (1982); G. Röpke, M. Schmidt, L. Münchow, and H. Schulz, Nucl. Phys. A399, $587(1982)$.

[3] P. Nozières and S. Schmitt-Rink, J. Low Temp. Phys. 59, 159 (1985).

[4] M. Schmidt, G. Röpke, and H. Schulz, Ann. Phys. (NY) 202, 57 (1990).

[5] R. Zimmermann, Many-Particle Theory of Highly Excited Semiconductors (Teubner, Leipzig, 1988).

[6] H. Stein, A. Schnell, T. Alm, and G. Röpke, Z. Phys. A 351, 295 (1995).

[7] A. Bohr and B. R. Mottelson Nuclear Structure (Benjamin, N.Y., 1969), Vol. I; P. Ring and P. Schuck, The Nuclear Many-Body Problem (Springer, N.Y., 1980).

[8] J. Dobaczewski, W. Nazarewicz, T. R. Werner, J. F. Berger, C. R. Chinn, and J. Dechargé, Phys. Rev. C 53, 2809 (1996).

[9] S. L. Shapiro and S. A. Teukolsky, Black Holes, White Dwarfs and Neutron Stars: The Physics of Compact Objects (Wiley, N.Y., 1983); D. Pines, R. Tamagaki, and S. Tsurata (eds.), Neutron Stars (Addison-Wesley, N.Y., 1992).

[10] T. Alm, G. Röpke, M. Schmidt, Z. Phys. A 337, 355 (1990); T. Alm, B. L. Friman, G. Röpke, and H. Schulz, Nucl. Phys. A551, 45 (1993); B. E. Vonderfecht, C. C. Gearhart, W. H. Dickhoff, A. Polls, and A. Ramos, Phys. Lett. B 253, 1 (1992); M. Baldo, I. Bombacci, and U. Lombardo, Phys. Lett. B 283, 8 (1993). 
[11] M. Baldo, U. Lombardo, and P. Schuck, Phys. Rev. C 52, 975 (1995).

[12] A. L. Goodman, Nucl. Phys. A352, 30 and 45 (1981); Nucl. Phys. A369, 365 (1981).

[13] M. Baldo, U. Lombardo, and P. Schuck, Phys. Rep. 242, 159 (1994).

[14] P. Danielewicz and P. Schuck, Nucl. Phys. A567, 78 (1994); S. Koh, Phys. Rev B 49, 8983 (1994); J. Dukelsky, G. Röpke, and P. Schuck, Nucl. Phys. A (to be published).

[15] V. G. J. Stoks et al., Phys. Rev. C 49, 2950 (1994); R. B. Wiringa et al., Phys. Rev. C 51, 38 (1995); R. Machleidt, F. Sammarruca, and Y. Song, Phys. Rev. C 53, 1483 (1996).

[16] P. Nozières and D. Saint James, J. Physique 43, 1133 (1982).

[17] J. G. Valatin and D. Butler, Nuovo Cimento 10, 37 (1958).

[18] M. Cauvin, V. Gillet, and F. Soulmagnon, Nucl. Phys. A361, 192 (1981); Y. K. Gambhir, P. Ring, and P. Schuck, Phys. Rev. Lett. 51, 1235 (1983); K. Varga, R. G. Lovas, and R. J. Liotta, Phys. Rev. Lett. 69, 37 (1992); F. Aldabe, G. G. Dussel, and H. M. Sofia, Phys. Rev. C 50, 1518 (1994).

[19] P. Schuck and K. Taruishi, Phys. Lett. B 385, 12 (1996).

[20] B. Buck, A. C. Merchant, and S. M. Perez, Phys. Rev. Lett. 72, 1326 (1994); B. Buck, J. C. Johnston, A. C. Merchant, and S. M. Perez, Phys. Rev. C 53, 2841 (1996).

[21] O. Dumitrescu, Phys. Rev. C 49, 1466 (1994).

[22] A. L. Ivanov, L. V. Keldysh and V. V. Panashchenko, Sov. Phys. JETP 72, 359 (1991).

[23] T. Alm, G. Chanfray, P. Schuck, and G. Welke, Nucl. Phys. A612, 472 (1997). 


\section{FIGURES}

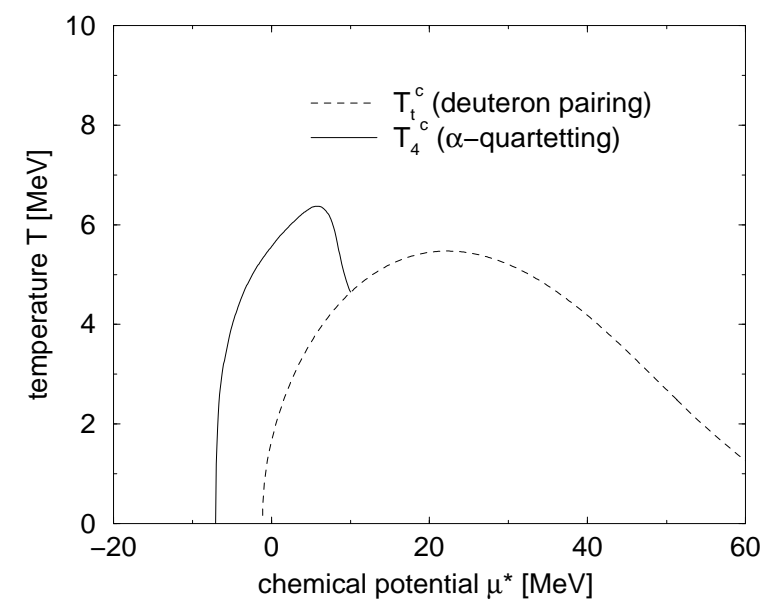

FIG. 1. Critical temperatures for the onset of quantum condensation in symmetric nuclear matter, model calculation. The critical temperature of the onset of two-particle pairing $T_{t}^{c}$ is compared with $T_{4}^{c}$ for the onset of a four-particle condensate, as a function of the chemical potential.

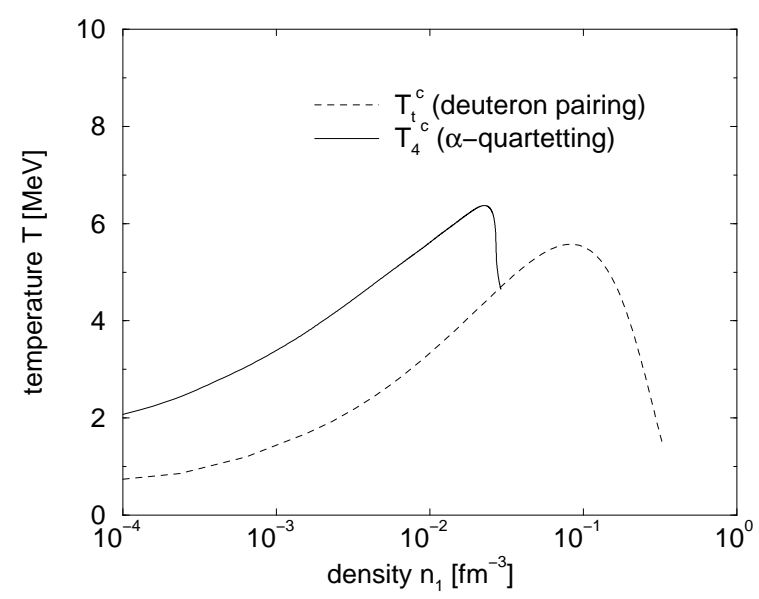

FIG. 2. The same as Fig. 1 but as a function of the uncorrelated density $n_{1}$. 